formulation of rod and frame performance in the schizophrenias. Perceptual \& Motor Skills. $1968.27 .1111-1114$.

SUGFRMAN. A. A.. \& CANCRO, R. Field independence and outcome in schizophrenia: A U-shaped relationship. Perceptual \& Motor Skills, 1968, 27, 1007-1013.

VAUGHT, $G$. M. Expected scores in the rod-and-frame test: Fuel for the
Immergluck-Pressey fire, Psychonomic Science, $1968,13,248$.

VAUGHT, G. M. Expected scores in the rod-and-frame test revisited. Psychonomic Science, $1970,18,111$.

WITKIN, H. A., LEWIS, H. B., HERTZMAN, M. MACHOVER, K., MEISSNER, P. B., \& WAPNER, S. Personality through perception. New York: Harper, 1954.

\title{
NLM strength and CVC-CVC response-time recall*
}

\author{
HOWARD I. THORSHEIM $\dagger$ \\ University of Illinois, Urbana, Ill. 61801
}

One hundred and twenty Ss were tested for the effect of natural language mediator (NLM) strength on the retention of NLMs and paired-associate response terms over 1 week. Hypotheses were that stronger NLMs would be retained best, as would the response terms of their pairs. At three different sessions, Ss (1) generated NLMs to $50 \mathrm{CVC}$ pairs, (2) learned 18 of the pairs by a drop-out procedure and had an immediate retention test, and (3) had a 1-week retention test. The main experimental group practiced the pairs along with their NLMs in Session 2, while two groups practiced only the pairs. Two amounts of practice in Session 2 was also a variable. Results were that (1) practice of pairs with their NLMs increased the frequency of NLM recall, (2) NLM and response term recall were both a function of amount of practice, and (3) presence of the NLM during Session 2 practice facilitated recall only in the immediate test.

Natural language mediators (NLMs) consist of idiosyncratic associations between new material to be learned and past language habits (Adams, 1967). Montague, Adams, \& Kiess (1966) established NLMs as a potent variable for the long-term retention of paired associates when the NLMs are remembered. However, they found that $73 \%$ of the NLMs formed in acquisition were forgotten by the time

\footnotetext{
*This experiment is based on a dissertation submitted to the Graduate College of the University of Illinois in partial fulfillment of the requirements for the $\mathrm{PhD}$ degree in psychology. The thesis was under the supervision of Jack A. Adams. The research was supported by Grant MH-12022 from the U.S. Public Health Service.

+ Now at St. Olaf College, Northfield, Minnesota 55057.
}

of a recall test $24 \mathrm{~h}$ later, and, if the NLM was forgotten, the probability was low that the criterion response would be recalled. Because NLMs appear to be a major factor in retention, and yet are themselves responses that are susceptible to forgetting, means of increasing the retention of NLMs must be found if an increase in the long-term retention of criterion responses is to be achieved.

The purpose of the present experiment was to examine the relationship of NLM strength to NLM retention and paired-associate response term retention. NLMs are responses, and their strength can be manipulated by practice and be indexed by their empirical probability during paired-associate learning and recall.

\section{PROCEDURE}

All $S s$ in the experiment underwent three separate sessions. In Session 1 they generated NLMs to $50 \mathrm{CVC}$ pairs of $26.0 \%$ mean associability as scaled by Montague \& Kiess (1968). Each $S$ was required to form a NLM for every pair and did so. In Session 2, Ss practiced $18 \mathrm{CVC}$ pairs to a criterion of either one $(\mathrm{C} 1)$ or three $(\mathrm{C} 3)$ correct recalls per item, followed by an immediate retention test. One week after Session 2, all Ss returned for Session 3, which was another retention test just like the one immediately following Session 2 . Three main treatment groups were involved. Treatment 1 (T1) was the experimental group that had NLMs present in acquisition for practice, and Treatments 2 and 3 (T2 and T3) to control for specific and nonspecific transfer effects from Session 1 to Session 2. Combined factorially with $\mathrm{T} 1, \mathrm{~T} 2$, and $\mathrm{T} 3$ were the two levels of the practice criterion, $\mathrm{C} 1$ and C3.

In detail, $40 \mathrm{Ss}$ in $\mathrm{T} 1$ each generated a NLM for each of 50 CVC pairs in Session 1. The time required to form a NLM ranged from 2 to $65 \mathrm{sec}$, but Ss were unlimited in the time they could take. For Session 2, which was held 1 week after Session 1, 18 CVC pairs were selected at random from the set of 50 pairs. In Session 2, the 18 pairs were practiced and learned along with their idiosyncratic NLMs from Session 1. Half of the T1 Ss had Practice Criterion $\mathrm{Cl}$ and half had Practice Criterion C3. Practice Criterion C1 consisted of one correct response recall per item in a drop-out procedure differing from that described by Battig (1965) only in that the NLM generated by each S in Session 1 was typed on the card along with the item for the study portion of the trial. Thus, practice was as follows: (1) All 18 items were presented one at a time together with the idiosyncratic NLM generated for each item by $S$ in Session 1, (2) next, only the stimulus terms of each pair were presented one at a time and $S$ was tested for his ability to spell the appropriate response term correctly, (3) items for which $\mathrm{S}$ correctly recalled the response term were dropped, (4) $S$ was then shown the balance of the items in the shortened deck of cards together with the corresponding NLMs, for another study opportunity, in a new random order. The Practice Criterion $\mathrm{Cl}$ was defined as being met when $S$ had correctly recalled each response term once after as many such study-test presentations as necessary had occurred. The Practice Criterion C3 consisted of repeating the procedure until three correct response recalls had occurred for each pair.

The immediate-retention test was given 
after acquisition to assess the availability of any NLMs and response retention, and served as a baseline for measuring NLM availability and response retention at a recall test 1 week later. The retention tests were the same for all Ss, with Ss being instructed at both recall tests to spell the response term for a pair and to say any NLM he may have used to learn the item during acquisition. Time allowed per card during the study and test portion of the trial was always $5 \mathrm{sec}$, and $15 \mathrm{sec}$ was allowed per card during immediate and 1 -week recall tests.

In order to rule out the alternative explanations of specific and nonspecific transfer effects from Session 1 to Session 2 as reasons for any effects observed, T2 and T3 served as the respective controls. The $40 \mathrm{Ss}$ in T2 underwent Session 1 exactly like $\mathrm{T} 1 \mathrm{Ss}$, with the same set of 50 pairs. In Session 2 they received the same subset of 18 pairs as the T1 Ss. Half of the Ss went to Practice Criterion $\mathrm{Cl}$ and half to C3. The difference between $\mathrm{T} 1$ and $\mathrm{T} 2$ was that T2 Ss did not have their Session 1 NLMs along with the pairs during the practice of Session 2. Session 3 was the same as for T1.

T3 was designed to control for nonspecific transfer effects from Session 1 for NLM formation, and any effects it might have on subsequent Sessions 2 and 3 . In Session 1, the $40 \mathrm{Ss}$ of T3 were given a different set of 50 pairs from those seen by $\mathrm{T} 1$ and T2 Ss, but the set was of $26.0 \%$ mean associability just like the set used for T1 and T2. T3 generated NLMs for their set of 50 pairs in the same procedure as that used for T1 and T2. In Session 2, each $S$ in T3 received the same subset of 18 items used by $T 1$ and T2 Ss, but no NLMs were provided with the pairs during acquisition. Thus T3 had the same amount of experience in generating NLMs as Ss in both $T 1$ and $T 2$, but the NLMs they generated in Session 1 were not provided nor would they have been directly relevant to the pairs of Session 2. Again, half of the Ss practiced to Criterion $\mathrm{C} 1$ and half to $\mathrm{C} 3$. Session 3 was as before.

In all treatments, responses were in writing for Session 1 and oral in Sessions 2 and 3.

\section{SUBJECTS AND APPARATUS}

Ss were 120 paid male and female volunteers from the University of Illinois. Twenty-two males and 98 females were randomly distributed among groups, with the constraint that the male/female ratio be the same in each group.

Presentation of materials in Session 1 was with a Carousel slide projector. For Sessions 2 and 3 , all materials were typed on $5 \times 8$ in. cards and presented manually to Ss by dealing the cards on a table. Ss
Table 1

Mean Number of NLMs Recalled

\begin{tabular}{lrrrrr}
\hline & \multicolumn{2}{c}{$\mathrm{C} 1$} & & \multicolumn{2}{c}{$\mathrm{C} 3$} \\
\cline { 2 - 3 } \cline { 5 - 6 } & Immediate & 1 Week & Immediate & 1 Week \\
\hline T1 & 13.05 & 11.65 & 16.20 & 13.85 \\
T2 & 9.30 & 8.65 & 11.85 & 9.75 \\
T3 & 5.65 & 3.65 & 8.70 & 5.50 \\
\hline
\end{tabular}

were tested individually. Timing of card presentations was controlled by the clicking of a relay actuated by Hunter interval timers.

\section{RESULTS}

Table 1 presents the mean number of NLMs remembered by groups at both recall tests. To assess the effect of the experimental variables upon the availability of any NLMs at recall, a 3 by 2 by 2 analysis of variance was conducted with the three treatments, two practice criteria, and two retention intervals. The cell score for $S$ was the number of items for which a NLM was remembered. Treatments $[F(2,114)=51.63], \quad$ practice $[F(1,114)=13.62]$, and retention interval $[F(1,114)=86.00]$ were all significant $(p<.01)$. Scheffé tests (Edwards, 1965. p. 131) indicated that $T 1, T 2$, and $T 3$ all differed significantly $(\mathrm{p}<.01)$ from one another in terms of number of NLMs, for both practice criteria, for both recall tests. A significant Practice by Retention Interval interaction $[\mathrm{F}(1,114)=8.14, \mathrm{p}<.01]$ was found by Scheffé tests to be due to significantly greater NLM forgetting for C3 compared to $\mathrm{C} 1$ even though $\mathrm{C} 3$ yielded more NLMs at both recall tests.

Table 2 presents the mean number of criterion responses recalled by groups at both recall tests, and it parallels the NLM data in Table 1. The basis for judging response correctness was that all letters of the response term be recalled, and in the correct order. A 3 by 2 by 2 analysis of variance was conducted parallel to that for the NLM data of Table 1. Treatments $[F(2,114)=15.68]$, practice $[F(1,114)=99.13]$, and retention interval $[F(1,114)=260.29]$ were all significant $(\mathrm{p}<.01)$. Scheffé tests indicated that $\mathrm{T} 1$ was superior to $\mathrm{T} 2$ only at the immediate test, whereas T3 was significantly poorer than $\mathrm{T} 1$ or $\mathrm{T} 2$ at both the immediate and 1-week tests.

\section{DISCUSSION}

If paired-associate items are presented for learning along with idiosyncratic NLMs

Table 2

Mean Number of Responses Correctly Recalled

$\mathrm{C} 1$

$\mathrm{C} 3$

\begin{tabular}{lccccc}
\hline & \multicolumn{2}{c}{$\mathrm{C1}$} & \multicolumn{2}{c}{$\mathrm{C3}$} \\
\cline { 5 - 6 } \cline { 5 - 6 } & Immediate & 1 Week & Immediate & 1 Week \\
\hline T1 & 8.55 & 4.85 & 15.35 & 9.15 \\
T2 & 6.45 & 4.25 & 14.00 & 8.20 \\
T3 & 5.35 & 2.20 & 10.50 & 5.70 \\
\hline
\end{tabular}

that Ss have formed previously for the pairs, the NLMs will be stronger and more available at later recall tests than if the NLMs are not present during learning. In addition, increasing the amount of practice with items whose NLMs are present increases NLM strength and their availability at recall.

Practice of pairs alone increased response recall, which agrees with previous findings on retention and degree of practice (Postman, 1962: Underwood \& Keppel, 1962), but response retention was greater when NLMs were practiced along with the pairs (as with Group T1) than when pairs were practiced alone (as with Group T2). The procedure for T 1 appeared to help $S$ utilize his past associations for pairs, which is support for the transfer-of-training role of NLMs in learning and recall (Adams, 1967: Montague, Adams, \& Kiess, 1966). The finding that Groups T1 and T2 differed significantly from one another in response retention only at the immediate test is puzzling. It has been shown that NLMs facilitate response recall only if the NLMs are remembered at the time of recall (Montague, Adams, \& Kiess, 1966), but in the present study the superiority of T1 over T2 in remembered NLMs was not reflected in superior response recall. A possible explanation, which requires further empirical test, is that it is not enough to look at simple availability of NLMs at recall. One may also need to ascertain whether or not $\mathrm{S}$ remembers how to use his NLM, i.e., whether S remembers the rule relating the NLM to the item such that the response term of the pair can be decoded from the NLM at time of recall. In other words, one may remember the NLM originally used to encode the paired associate, but over time forget the rule necessary to decode the criterion response from the NLM.

\section{REFERENCES}

ADAMS, J. A. Human memony. New York: McGraw-Hill, 1967

BATTIG, W. F. Procedural problems in paired-associate learning research. Psychonomic Monograph Supplements, 1965, 1, 1-12.

EDWARDS, E. L. Experimental design in psychological research. New York: Holt, Rinehart, \& Winston, 1965.

MONTAGUE, W. E., ADAMS, J. A., \& KIESS, H. $O$. Forgetting and natural language mediation. Journal of Experimental Psychology, 1966, $72,829-833$.

MONTAGUE, W. E., \& KIESS, H. O. The associability of CVC pairs. Journal of Experimental Psychology Monograph Supplement, 1968, 78, Part 2.

POSTMAN, L. Repetition and paired-associate learning. American Journal of Psychology, $1962,75,372-389$.

UNDERWOOD, B, J., \& KEPPEL, G. One-trial learning. Journal of Verbal Learning \& Verbal Behavior, 1962, 1, 1-13. 